\title{
COMPUTER VISION AND NEURAL NETWORKS FOR LIBRAS RECOGNITION
}

\author{
Silas Luiz Furtado ${ }^{1}$, Jauvane de Oliveira ${ }^{2}$ \\ ${ }^{1}$ Instituto Militar de engenharia -SE/8 - silasfurtado@outlook.com.br \\ ${ }^{2}$ Mathematical and computational methods department, National \\ Laboratory for Scientific Computing - LNCC - jauvane@acm.org \\ Rio de janeiro, Brasil
}

\begin{abstract}
In recent years, one can find several efforts to increase the inclusion of people with some type of disability. As a result, the global study of sign language has become an important research area. Therefore, this project aims at developing an information system for the automatic recognition of the Brazilian Sign Language (LIBRAS). The recognition shall be done through the processing of videos, without relying on support hardware. Given the great difficulty of creating a system for this purpose, an approach was developed by dividing the process into stages. In addition to dynamically identifying signs and context, neural network concepts and tools were used to extract the characteristics of interest and classify them accordingly. In addition, a dataset of signs, referring to the alphabet in LIBRAS, was built as well as a tool to interpret, with the aid of a webcam, the signal executed by a user, transcribing it on the screen.
\end{abstract}

Index Terms-LIBRAS, inclusion, disabilities, computer vision.

\section{IntRodução}

Não podemos ignorar que ao nosso redor, todos os dias milhares de pessoas enfrentam diversos obstáculos devido a deficiências de diversas naturezas. Dentre todas essas naturezas, podemos destacar a deficiência auditiva, quando o seu portador possui uma severa dificuldade em se comunicar em uma sociedade cuja comunicação se faz predominantemente por meios sonoros.

Dentre as deficiências existentes, de acordo com a organização mundial de saúde [1] cerca de $5 \%$ da população mundial, ou seja, por volta de 466 milhões de pessoas no mundo, possuem surdez incapacitante. Isso significa que apenas essa parcela de $5 \%$ possui perda auditiva maior que $40 \mathrm{~dB}$ quando falamos do indivíduo adulto e $30 \mathrm{~dB}$ nas crianças, englobando tipos de suave a severo, e que a estimativa é de que até o ano de 2050 este número aumente para cerca de $10 \%$ da população.

Olhando os números da realidade brasileira, segundo o último censo realizado em 2010 pelo IBGE [2] cerca de 10 milhões de brasileiros possuem deficiência auditiva, representando cerca de $5,1 \%$ da população total brasileira. Deste montante, cerca de 2 milhões de pessoas possuem deficiência severa considerando 344,2 mil não escutam por completo e 1,7 milhões têm grande dificuldade para escutar.

Para a maioria dessas pessoas, é de extrema dificuldade utilizar a língua portuguesa devido a não conseguir assimilar e replicar a oralidade da língua. Com isso é necessário que eles se utilizem de uma língua visual-espacial para se comunicar, sendo LIBRAS a principal língua utilizada por deficientes auditivos.

Uma grande dificuldade no reconhecimento de LIBRAS é a quantidade de parâmetros que são considerados para que um sinal possua algum significado. De acordo com Stokoe [3] um sinal com algum significado é formado combinando cinco parâmetros: (a) configuração de mão; (b) ponto de articulação; (c) movimento; (d) orientação/direção; e (e) expressões não manuais.

Além disso, apesar de existir configurações de mão representando todas as letras do alfabeto, na comunicação diária não é comum serem utilizados essas configurações e sim gestos referentes a verbetes para formação de sentenças. Nesse caso, na segmentação das imagens para que um gesto seja reconhecido é necessário uma grande capacidade computacional.

Este trabalho inspira-se em trabalhos prévios [4] e, através do uso de visão computacional, aplica uma metodologia e a mostra através de um protótipo capaz de, com o auxílio de uma webcam, analisar e reconhecer sinais do alfabeto de LIBRAS, traduzindo automaticamente para a língua portuguesa e transcrevendo interativamente seu significado na tela do computador.

\section{Metodologia Para o sistema e conceitos}

Para facilitar o entendimento, serão apresentadas as etapas principais do sistema de maneira modular. Cada etapa da metodologia adotada corresponde a um módulo do sistema, e essas etapas seguirão o fluxo de execução do sistema apresentado na Figura 1.

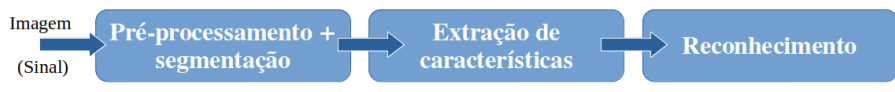

Fig. 1. Metodologia adotada.

$\mathrm{Na}$ concepção inicial do trabalho encontramos apenas datasets públicos de expressões em LIBRAS, porém mesmo sendo públicos estes datasets ora não estavam em formatos amigáveis para o uso em nosso trabalho, ora precisavam de aprovação da instituição detentora dos arquivos e as mesmas não responderam as solicitações. Por isso, para desenvolver e testar o sistema de reconhecimento de LIBRAS, optamos 
então, por construir o próprio dataset baseado em quadros extraídos de vídeos.

Os vídeos foram gravados no próprio ambiente de desenvolvimento, sendo assim um ambiente com pouco controle. Dada as circunstâncias, decidiu-se escolher o alfabeto como ponto de partida, por ser a base de qualquer linguagem e por ter características suficientes para representar o problema, já que mesmo neste conjunto pequeno de sinais pode-se notar variedade de gestos com as mãos e semelhança entre alguns sinais. É possível destacar por exemplo, a semelhança entre os sinais referentes as letras $\mathrm{A}$ e $\mathrm{E}$, ou as letras $\mathrm{M}$ e $\mathrm{N}$, como mostra a Figura 2.

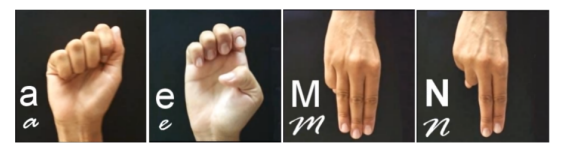

Fig. 2. Representações das letras A,E,M e N.

Com isso, as gravações resultaram em oitenta e sete mil frames referentes a todas as letras do alfabeto junto com dois sinais de controle, sendo um para significar possíveis espaços entre caracteres e o outro para deletar caracteres. Além destes incluiu-se também imagens com ausência de sinais, ou seja, imagens que representam o fundo.

Durante o desenvolvimento deste trabalho, foram procurados bancos de imagens públicos, que contivessem sequências de imagens (vídeos) relacionadas ao alfabeto de LIBRAS, mas nenhum banco público foi encontrado com essas características, sendo por isso necessária esta etapa.

\section{A. Sobre o pré-processamento}

Como o objetivo final é realizar o mínimo de interferência computacional nas etapas iniciais do processo, foi assegurado apenas que a amostra tentasse representar o máximo possível a realidade. Ou seja, a aquisição das imagens se deu em ambientes com luminosidade diversificada.

Como o interesse está apenas nas configurações de mão, os frames limitaram-se apenas à região das mãos. Em seguida foi realizada a retirada de fundo e após a conclusão dessa etapa, foi realizada a extração de características.

\section{B. Remoção do fundo da imagem}

Para a remoção do fundo da imagem, inicialmente foi realizada a conversão do espaço de cores original para outro. No caso de uma transformação para o espaço de cores RGB, a ordem dos canais deve ser especificada explicitamente (RGB ou BGR). Para esta tarefa utilizou-se a biblioteca OpenCV. O formato de cor padrão no OpenCV é geralmente chamado de RGB, mas na verdade é BGR (os bytes são invertidos).

Portanto, o primeiro byte em uma imagem colorida padrão (24 bits) será um componente azul de 8 bits, o segundo byte será verde e o terceiro byte será o componente vermelho. $\mathrm{O}$ quarto, quinto e sexto bytes seriam então o segundo pixel (azul, depois verde e depois vermelho) e assim por diante.

As faixas convencionais para os valores dos canais $R, G$ e B são:
- 0 a 255 para CV_8U imagens

- 0 a 65535 para $\mathrm{CV}_{-} 16 \mathrm{U}$ imagens

- 0 a 1 para CV_32F imagens

No caso de transformações lineares, o intervalo não importa. Porém, no caso de uma transformação não linear, uma imagem RGB de entrada deve ser normalizada no intervalo de valores apropriado para obter os resultados corretos. Se esta função for utilizada em imagens de 8 bits, a conversão terá algumas informações perdidas. Para muitos aplicativos, isso não será perceptível, mas é recomendável usar imagens de 32 bits em aplicativos que precisam de toda a gama de cores ou que convertam uma imagem antes de uma operação e depois convertam novamente.

Então a transformação de RGB para escala de cinza pode ser representada da seguinte forma:

$$
Y=0.299 * R+0.587 * G+0.114 * B
$$

Após esta etapa é aplicado um filtro gaussiano. No processamento de imagens o filtro gaussiano (também conhecido como suavização gaussiana) é o resultado do desfoque de uma imagem por uma função gaussiana, que é muito utilizado para a redução de ruídos de uma imagem, ou seja, com ele busca-se aprimorar as estruturas de imagem em diferentes escalas.

Matematicamente, este filtro utiliza uma função gaussiana, que expressa a distribuição normal no campo da estatística para calcular a transformação a ser aplicada em cada pixel da imagem de entrada. Em duas dimensões podemos expressar um gaussiano da seguinte forma:

$$
G_{0}(x, y)=A e^{\frac{-\left(x-\mu_{x}\right)^{2}}{2 \sigma_{x}^{2}}+\frac{-\left(y-\mu_{y}\right)^{2}}{2 \sigma_{y}^{2}}}
$$

Onde $\mathrm{x}$ é a distância da origem no eixo horizontal, y é a distância da origem no eixo vertical e $\sigma$ é o desvio padrão da distribuição gaussiana. Quando aplicada em duas dimensões, essa fórmula produz uma superfície cujos contornos são círculos concêntricos com uma distribuição gaussiana a partir do ponto central. Os valores dessa distribuição são usados para construir uma matriz de convolução aplicada à imagem original.

O novo valor de cada pixel é definido como uma média ponderada da vizinhança desse pixel. $\mathrm{O}$ valor do pixel original recebe o peso mais significativo (com o valor gaussiano mais alto) e os pixels vizinhos recebem pesos menores à medida que aumenta a distância do pixel original. Isso resulta em um desfoque que preserva limites e bordas melhor que outros filtros de desfoque mais uniformes, como ilustrado visualmente na Figura 3.

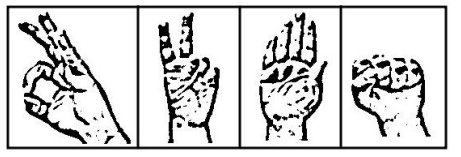

Fig. 3. Filtro gaussiano com threshold. 


\section{EXTRAÇÃO DE CARACTERÍSTICAS}

Uma rede neural convolucional pode ser dividida em dois blocos, o primeiro bloco é responsável pela extração de características e o segundo, pela classificação. Naquela encontramse as camadas de convolução e as camadas de pooling, onde se encontra a camada fully-connected.

Matematicamente, uma convolução é uma operação linear que a partir de duas funções, gera uma terceira (chamada de feature map). No contexto de imagens, a camada de convolução é composta por diversos neurônios, onde cada um é responsável por aplicar um filtro, e este filtro é chamado de kernel, que é uma matriz de convolução responsável para a detecção de bordas, características e traços importantes na imagem. Cada matriz, possui valores dos pesos sinápticos da rede e ao percorrer toda a imagem, é gerado uma nova imagem com o nome de mapa de características.

O salto é a quantidade de colunas/linhas de pixels que o kernel deve 'saltar' ao percorrer a imagem. O preenchimento de zero serve para que as camadas não diminuam muito mais rápido do que é necessário para o aprendizado. Para entender para que ele serve, imagine que enquanto o kernel varre a imagem pulando os pixels de acordo com o valor do salto, possivelmente, pelos valores de tamanho de cada um, alguma coluna ou linha de pixel será perdida e não será analisada. O padding adiciona borda na imagem com valores 0 para não ter interferência no resultado da convolução e o kernel poder analisar toda a imagem.

Quando se está criando a estrutura da rede, na convolução é necessário passar alguns parâmetros e inicialmente existem 3 parâmetros importantes para serem usados. São eles o tamanho da matriz do kernel, o salto (stride) e o preenchimento de zero (zero padding).

Após uma camada de convolução ainda no bloco de extração de características, pode-se aplicar uma camada de pooling. Essa camada ajuda a diminuir a quantidade de parâmetros para a próxima camada de convolução ou para as camadas totalmente conectadas. A camada de pooling serve para simplificar a informação e reduzir a dimensionalidade da convolução. Essa redução é importante por questão de agilidade no treinamento.

$\mathrm{O}$ pooling também possui uma matriz que percorre o mapa de características gerado pela convolução e prepara um mapa de características condensadas. Possui alguns métodos de ativação que podem ser aplicados, onde os mais utilizados são Max Pooling e Average Pooling. Cada neurônio da camada de pooling pode resumir uma região de neurônios da camada de convolução. Utilizando a função de max pooling, por exemplo, um neurônio gera a ativação máxima na região aplicada.

1) Transferência de aprendizado: A transferência de aprendizado é um problema no campo de aprendizado de máquina que consiste em armazenar o conhecimento adquirido ao resolver um problema e aplicá-lo a um outro diferente, porém de mesma essência. Do ponto de vista prático, a transferência de informações de tarefas aprendidas para o aprendizado de novas tarefas tem o potencial de melhorar significantemente a eficiência da amostra de um agente de aprendizagem por reforço.
Como este trabalho, consiste em uma classificação de imagem, no estudo comparativo dentre os modelos mais utilizados realizado por Bianco et al. [5], o modelo Inception é um dos que possui boa acurácia com pouco custo computacional para utilizá-lo, como mostra a Figura 4.

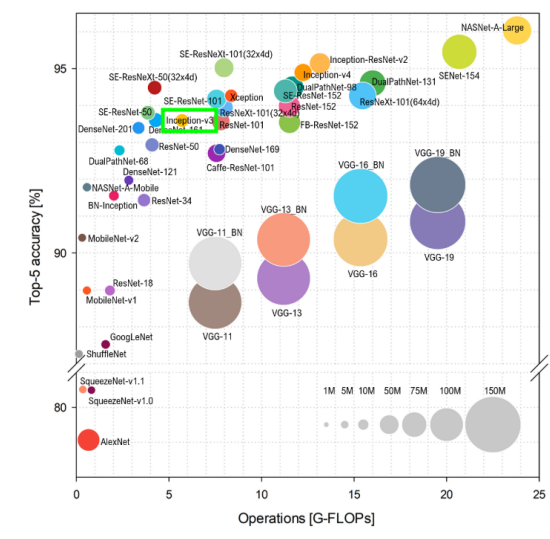

Fig. 4. Comparativo entre arquiteturas. Fonte: Bianco et al.,2018

2) Rede Inception: A rede neural Inception foi desenvolvida com base na arquitetura GoogleNet vista em [6]. Tal abordagem também teve inspiração na abordagem baseada no córtex visual dos primatas, como relatado por Serre et al. [7] que pode lidar com detecção de objetos em várias situações. Um dos critérios importantes da arquitetura Inception é a adaptação da abordagem "Network in Network" descrita em Lin et al. [8], o que aumenta o poder representacional das redes neurais. Isso também possibilitou melhoras em possíveis gargalos computacionais por redução de dimensão para convoluções $1 \times 1$.

Nesse contexto foi desenvolvido o modelo Inception v3 [9], utilizado neste trabalho, por ser utilizado amplamente no reconhecimento de imagem. Este modelo foi testado na base de dados ImageNet, que consiste em uma vasta base de imagens organizadas hierarquicamente. Nela cada nó da hierarquia é composto por milhares de imagens. Nessa base de dados, esse modelo alcançou uma precisão superior a 78\%. Esse modelo é o auge de muitas ideias desenvolvidas por vários pesquisadores ao longo de vários anos. O modelo é composto basicamente por componentes simétricos e assimétricos, incluindo convoluções, agrupamentos médios, agrupamentos máximos, concatenações, desistências e camadas totalmente conectadas, como podemos observar na Figura 5.

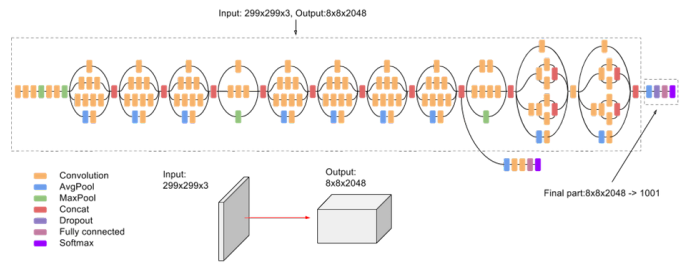

Fig. 5. Arquitetura Inception v3. 
Como este trabalho utiliza a transferência de aprendizado, as configurações para esses passos foram definidas pela arquitetura adotada (InceptionV3). Para cada magnitude foram gerados os vetores de características com base no modelo interno de extração de características da arquitetura utilizada.

A figura 6 mostra a camada de extração de características da arquitetura utilizada.

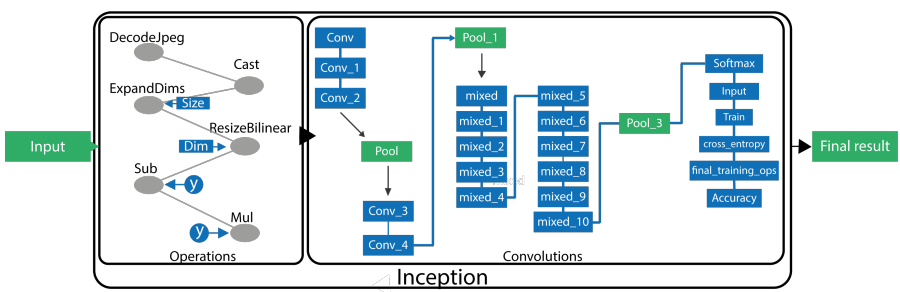

Fig. 6. Gráfico gerado pela arquitetura utilizada.

\section{RECONHECIMENTO}

A função de ativação Softmax é utilizada nessa etapa por ser uma função de classificação que deve ser aplicada na camada de saída da rede. Esta função também é um tipo de função sigmóide, mas é útil nos problemas de classificação. Esta função pode ser definida como:

$$
\sigma(z)_{j}=\frac{e^{z_{j}}}{\sum_{k=1}^{K} e^{z_{k}}}
$$

A vantagem do uso da softmax é o intervalo de probabilidade de saída com valores entre 0 e 1 . Por exemplo, para as saídas como [1.2, 0.9, 0.75] quando aplicada a função softmax, obtêm-se [0.42, 0.31, 0.27] e isso essencialmente dá a probabilidade de identificar a qual classe pertence a imagem de entrada. Como o objetivo é gerar as probabilidades para definir a classe de cada entrada, esta foi a função escolhida.

\section{RESUltados DO TREINAMENTO DA REDE NEURAL E INTERFACE FINAL}

Como citado anteriormente, o experimento utilizou uma base de imagens do alfabeto em LIBRAS, somando-se a dois sinais de controle (uma para deleção e outro para representar um espaço).

Para a realização dos testes foram selecionadas aleatoriamente $70 \%$ da amostra para treino e $30 \%$ para teste, de forma semelhante ao que foi feito por Spanhol [10]. Durante esta fase, para que fosse possível definir um critério de parada, observou-se os valores de acurácia e de cross-entropy, tanto no conjunto de treino, quanto no de teste. Após atingir o número de 30.000 steps, foi possível observar os seguintes resultados de treino e teste, como mostra a Figura 7.

Para definir o melhor resultado deve-se observar os valores de acurácia e cross-validation em conjunto. Como pode-se notar, o melhor resultado ocorreu com 25.000 steps, como mostra a Tabela I:

A matriz de confusão para este trabalho pode ser verificada na Figura 8. Porém Quando estamos lidando com muitas

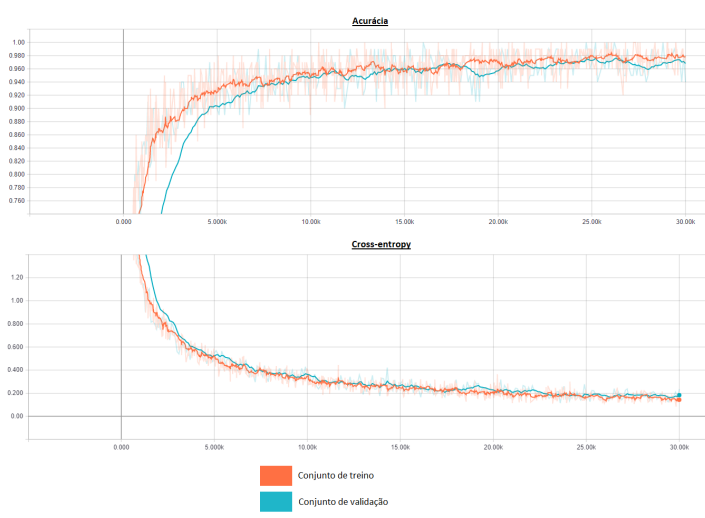

Fig. 7. Gráfico de acurácia e cross-validation nos conjuntos de treino e validação.

TABLE I

QUADRO DE TREINO E TESTE

\begin{tabular}{c|c|c|c|c}
\hline steps & Acc./treino & Acc./valid. & cross-ent./treino & cross-ent./valid. \\
\hline \hline 10.000 & 0.89 & 0.96 & 0.3853 & 0.3434 \\
15.000 & 0.97 & 0.98 & 0.2267 & 0.2801 \\
20.000 & 0.97 & 0.96 & 0.1776 & 0.2209 \\
25.000 & 0.99 & 0.97 & 0.1446 & 0.1813 \\
30.000 & 0.98 & 0.94 & 0.1240 & 0.2347 \\
\hline
\end{tabular}

classes é difícil apenas verificar a matriz de confusão devido a dificuldade de visualização. Por esse motivo podemos verificar outras métricas para avaliar o modelo treinado.

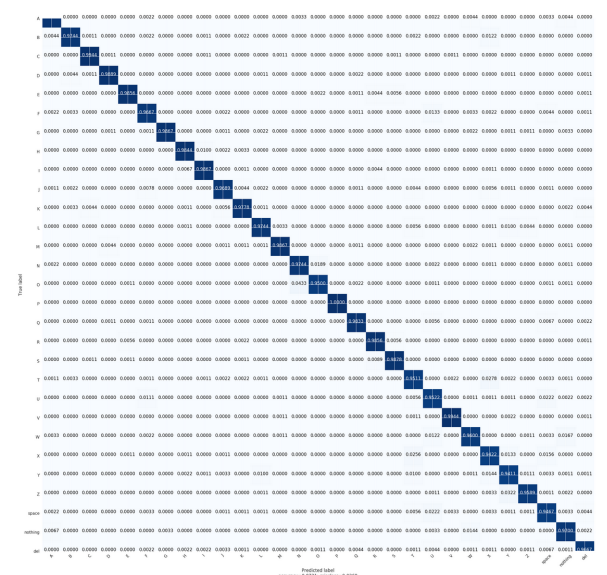

Fig. 8. Matriz de confusão.

As métricas de precisão, recall, $f 1$ score e especificidade para cada classe de nosso modelo são encontrados na Figura 9.

\section{A. Interface}

Para realizar o experimento de maneira interativa o desenvolvimento da ferramenta foi feito na linguagem python, utilizando o ambiente integral de desenvolvimento da empresa jetbrains chamada pycharm. Essa escolha foi feita por conta 


$\begin{array}{rrrrr} & \text { precision } & \text { recall } & \text { f1-score } & \text { support } \\ \text { A } & 0.98 & 0.98 & 0.98 & 900 \\ \text { B } & 0.98 & 0.97 & 0.98 & 900 \\ \text { C } & 0.99 & 0.99 & 0.99 & 900 \\ \text { D } & 0.99 & 0.99 & 0.99 & 900 \\ \text { E } & 0.99 & 0.99 & 0.99 & 900 \\ \text { F } & 0.97 & 0.97 & 0.97 & 900 \\ \text { G } & 1.00 & 0.99 & 0.99 & 900 \\ \text { H } & 0.99 & 0.98 & 0.98 & 900 \\ \text { I } & 0.98 & 0.99 & 0.99 & 900 \\ \text { J } & 0.98 & 0.97 & 0.97 & 900 \\ \text { K } & 0.98 & 0.98 & 0.98 & 900 \\ \text { L } & 0.98 & 0.97 & 0.98 & 900 \\ \text { M } & 0.99 & 0.99 & 0.99 & 900 \\ \text { N } & 0.95 & 0.97 & 0.96 & 900 \\ \text { O } & 0.98 & 0.95 & 0.96 & 900 \\ \text { P } & 1.00 & 1.00 & 1.00 & 900 \\ \text { Q } & 0.99 & 0.98 & 0.98 & 900 \\ \text { R } & 0.98 & 0.99 & 0.98 & 900 \\ \text { S } & 0.99 & 0.99 & 0.99 & 900 \\ \text { T } & 0.94 & 0.95 & 0.95 & 900 \\ \text { U } & 0.93 & 0.95 & 0.94 & 900 \\ \text { V } & 0.99 & 0.99 & 0.99 & 900 \\ \text { W } & 0.97 & 0.96 & 0.96 & 900 \\ \text { X } & 0.93 & 0.94 & 0.93 & 900 \\ \text { Y } & 0.93 & 0.94 & 0.94 & 900 \\ \text { Z } & 0.98 & 0.96 & 0.97 & 900 \\ \text { space } & 0.93 & 0.95 & 0.94 & 900 \\ \text { nothing } & 0.96 & 0.97 & 0.96 & 900 \\ \text { del } & 0.98 & 0.97 & 0.97 & 900 \\ & & & & \\ \text { accuracy } & & & 0.97 & 26100 \\ \text { macro avg } & 0.97 & 0.97 & 0.97 & 26100 \\ \text { weighted avg } & 0.97 & 0.97 & 0.97 & 26100\end{array}$

Fig. 9. Métricas de avaliação do modelo.

da ampla documentação da linguagem, além de ser linguagem amplamente utilizada por desenvolvedores em problemas de visão computacional e aprendizado de máquina.

$\mathrm{Na}$ construção também utlizamos a biblioteca OpenCV (Open Source Computer Vision), que consiste em uma biblioteca de domínio público, para desenvolvimento de algoritmos de visão computacional e aprendizado de máquina, licenciada pela licença BSD e amplamente utilizada por empresas e grupos de pesquisa. Esta biblioteca suporta diversas interfaces como C++, C, Python, Java e Matlab, podendo ser utilizado em praticamente todos os sistemas operacionais disponíveis no mercado.

A biblioteca possui módulos de processamento de imagens e vídeos, estrutura de dados, álgebra linear, interface gráfica para o usuário (GUI), controle de mouse e teclado, além de mais de 2.500 algoritmos, muitos dos quais considerados estado da arte, tais como os de segmentação, reconhecimento de faces, aprendizado de máquina, filtragem de imagens, rastreamento de movimento, entre outros.

O sistema captura a imagem com o auxílio de uma webcam e ao reconhecer um símbolo por três quadros consecutivos com uma confiança superior a $80 \%$, ele o transcreve em tela, como mostra a Figura 10.

Com base na revisão sistemática realizada anteriormente, reunimos em um quadro comparativo os trabalhos julgados importantes e com objetivos similares quanto à abordagem adotada.

Da Figura 11, verifica-se os trabalhos onde se encontram os melhores resultados em acurácia, que em sua maioria utilizaram algum dispositivo auxiliar. Além do mais, constatase que dentre as abordagens em LIBRAS, este trabalho atingiu um desempenho superior aos outros métodos mostrados, mesmo com menor poder computacional devido ao uso de redes neurais convolucionais.

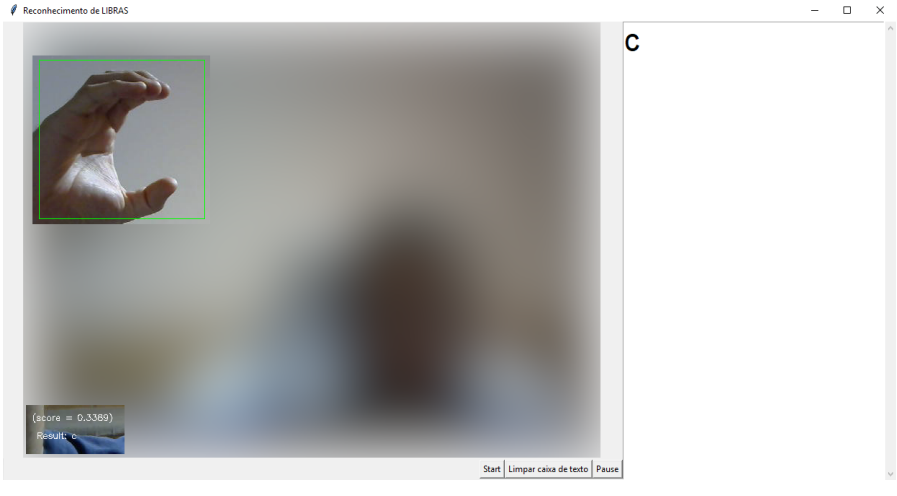

Fig. 10. Interface final construída.

\begin{tabular}{|c|c|c|c|c|c|}
\hline Referência & $\begin{array}{l}\text { Método de } \\
\text { aquisição }\end{array}$ & Idioma & Classificaçāo & Acurácia & Interativo \\
\hline Assalen [11] & \begin{tabular}{|l} 
Videos em atta \\
resoluçāo \\
.
\end{tabular} & Árabe & \begin{tabular}{|l|} 
Naive bayes \\
\end{tabular} & $99 \%$ & Nāo \\
\hline Sandjaja [12] & $\begin{array}{l}\begin{array}{l}\text { Videos com } \\
\text { luvas }\end{array} \\
\text { lut }\end{array}$ & Filipino & нмм & $85.52 \%$ & Nāo \\
\hline Highway [13] & \begin{tabular}{|c|} 
Videos comm \\
ropapa fundo \\
controlados
\end{tabular} & Australiano & HMM & $97 \%$ & Nāo \\
\hline Starner [14] & $\begin{array}{c}\begin{array}{l}\text { Videos com } \\
\text { luvas }\end{array} \\
\text { lat }\end{array}$ & Americano & нмм & $99.2 \%$ & Sim \\
\hline Isaacs [15] & $\begin{array}{l}\text { Videos em } \\
\text { estudiolo }\end{array}$ & Americano & ANN & $99.9 \%$ & Nāo \\
\hline Paulraj [16] & \begin{tabular}{|c|} 
Videos com \\
roupa ef fundo \\
controlados \\
\end{tabular} & Kod (Malasya) & ANN & $81.07 \%$ & Não \\
\hline Paulraj [17] & \begin{tabular}{|c} 
Viveos com \\
ropupa fundo \\
controlados \\
\end{tabular} & Americano & ANN & $92.07 \%$ & Nāo \\
\hline Quan [18] & \begin{tabular}{|l|} 
Videos em \\
fundo branco
\end{tabular} & Chinês & SVM & $95.55 \%$ & Nāo \\
\hline Madeo [19] & \begin{tabular}{|c}
$\begin{array}{c}\text { Videos com } \\
\text { roupa a tundo } \\
\text { controlados }\end{array}$ \\
\end{tabular} & LIBRAS & Comitê & $91.72 \%$ & Nāo \\
\hline Monteiro [20] & $\begin{array}{l}\text { Videos de } \\
\text { verbetes }\end{array}$ & LIBRAS & $k-n n$ & $75 \%$ & Nầo \\
\hline Padovani [21] & 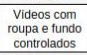 & LIBRAS & HмM & $80.1 \%$ & Nāo \\
\hline Gonçalves [22] & $\begin{array}{c}\text { Videos com } \\
\text { luvas }\end{array}$ & LIBRAS & Fuzzy & $84.3 \%$ & Sim \\
\hline Este trabalho & \begin{tabular}{|l|}
$\begin{array}{c}\text { Frames de } \\
\text { videos }\end{array}$ \\
\end{tabular} & LIBRAS & CNN & $97 \%$ & sim \\
\hline
\end{tabular}

Fig. 11. Quadro comparativo.

Este desempenho também se deve à técnica de transferência de aprendizado a partir de Deep learning treinada para o reconhecimento do conjunto ImageNet, pois a profundidade destas redes influencia nos resultados obtidos por classificadores, que receberam como dado de treino os vetores de características gerados a partir da extração feita por essas redes neurais previamente treinadas.

\section{CONClusão}

O objetivo deste trabalho foi o desenvolvimento de um sistema interativo para reconhecimento de LIBRAS, capaz de, com o auxílio de uma webcam, reconhecer sinais expressos e transcrever a palavra correspondente em língua portuguesa, isso sem a necessidade de auxílio de dispositivos eletromecânicos ou qualquer aparato junto ao corpo do usuário. Desta maneira pretende-se facilitar a comunicação entre surdos e/ou deficientes auditivos e pessoas que não conhecem uma língua gestual, proporcionando uma maior inclusão daqueles com o restante da sociedade. Foi realizada uma revisão sistemática, onde foi feita a comparação entre as abordagens adotadas nos trabalhos julgados importantes da área do presente trabalho e a abordagem adotada. Constatouse que dentre as abordagens em LIBRAS, este trabalho atingiu um desempenho superior aos outros métodos. 
O uso de redes neurais convolucionais como também a aplicação de Deep learning para a transferência de aprendizado, nos permite concluir que a transferência de conhecimento é uma boa técnica a ser explorada, pois apresentou bons resultados em comparação aos demais estudos. Quanto mais se preservar os dados das imagens originais, como é o caso da rede InceptionV3 que tem como entrada maiores dimensões de imagem, melhores são os resultados.

No desenvolvimento deste trabalho algumas dificuldades foram encontradas, como a ausência de um banco público de vídeos com execução do alfabeto em LIBRAS, limitando assim o tamanho do dataset utilizado no desenvolvimento do trabalho. Além disso, ao focar em trabalhos específicos para a língua portuguesa, praticamente todos se utilizaram da abordagem visual com restrições mecânicas, e/ou não se tratavam de sistemas interativos.

Apesar dos percalços encontrados, o objetivo de criar um sistema interativo com baixo custo computacional foi alcançado, sendo possível através do protótipo criar palavras a partir do alfabeto treinado, sem a necessidade de nenhum aparato auxiliar instalado no corpo do usuário. Acredita-se que todas as etapas do sistema proposto são contribuições para trabalhos futuros da área de reconhecimento de sinais, ou até mesmo trabalhos envolvendo processamento de imagens.

Devido ao tempo, complexidade do assunto em questão e a problemas enfrentados durante o desenvolvimento do trabalho, não era objetivo que o sistema desenvolvido fosse uma solução definitiva para reconhecimento de LIBRAS, e sim uma ferramenta para iniciar um estudo e ser aprimorado em trabalhos futuros.

Como trabalhos futuros, é sugerido se valer do desempenho da transferência de conhecimento feita a partir de bases de dados correlatas, e utilizar as técnicas de fine-tunning e data augmentation a fim de melhorar os modelos gerados pelas redes profundas.

Além disso, os testes realizados no sistema proposto utilizaram apenas os sinais referentes às letras do alfabeto em LIBRAS. Sendo assim, recomenda-se expandir o conjunto de palavras, assim como a diversificação dos executantes dos sinais. Outra vertente do trabalho seria tornar o sistema desenvolvido em uma aplicação mobile, possibilitando o uso do mesmo em qualquer dispositivo móvel.

Por fim, este trabalho focou nos parâmetros de configuração de mão, movimento e orientação para o reconhecimento dos sinais. Uma outra extensão, pouco tratada nos trabalhos relacionados e tampouco se encontra na literatura, seria as expressões não manuais como parâmetro para a composição dos sinais.

\section{REFERENCES}

[1] World Health Organization, "Deafness and Hearing Loss",pp. 1-4, 2018.

[2] da Silva Borges, And réa and Aparecida de Araujo Xavier, Eliane and Bercovich, Alicia,"Censo demográfico 2010",vol. 27,2011.

[3] Stokoe, William C. and Marschark, Marc,"Sign language structure: An outline of the visual communication systems of the american deaf", Journal of Deaf Studies and Deaf Education, pp. 3-37, vol. 10, 2005.
[4] Digiampietri, Luciano A. and Teodoro, Beatriz and Santiago, Caio R.N. and Oliveira, Guilherme A. and Araujo, Jonatas C.,'Um Sistema de Informação Extensível Para o Reconhecimento Automático de LIBRAS", VIII Simpósio Brasileiro de Sistemas de Informação (SBSI 2012), pp. 456-467, 2012.

[5] Bianco, Simone and Cadene, Remi and Celona, Luigi and Napoletano, Paolo,"Benchmark analysis of representative deep neural network architectures", IEEE Access,pp. 64270-64277, vol. 6, 2018.

[6] Christian Szegedy et al.,"Going Deeper with Convolutions", University of Michigan,pp. 163-182,2019.

[7] Thomas Serre, Lior Wolf, Stanley Bileschi, Maximilian Riesenhuber, and Tomaso Poggio,"Robust Object Recognition with CortexLike Mechanisms", IEEE transactions on pattern analysis and machine intelligence,pp.102-104,vol.38,2016.

[8] Lin, Min and Chen, Qiang and Yan, Shuicheng,"Network in network",2nd International Conference on Learning Representations, ICLR 2014 - Conference Track Proceedings,pp. 1-10,2014.

[9] Szegedy, Christian and Vanhoucke, Vincent and Ioffe, Sergey and Shlens, Jon and Wojna, Zbigniew,'Rethinking the Inception Architecture for Computer Vision",Proceedings of the IEEE Computer Society Conference on Computer Vision and Pattern Recognition, pp.28182826,vol.2016,2016.

[10] Spanhol, Fabio A. and Oliveira, Luiz S. and Petitjean, Caroline and Heutte, Laurent,"A Dataset for Breast Cancer Histopathological Image Classification",IEEE Transactions on Biomedical Engineering,pp. 14551462,vol. 63,2016.

[11] Assaleh, Khaled and Shanableh, Tamer and Fanaswala, Mustafa and Bajaj, Harish and Amin, Farnaz, "Vision-based system for continuous arabic sign language recognition in user dependent mode", pp. 19-27, vol. 2010, 2008

[12] Sandjaja, Iwan Njoto and Marcos, Nelson,'Sign language number recognition", NCM 2009 - 5th International Joint Conference on INC, IMS, and IDC,pp. 1503-1508, 2009.

[13] Goh, Paul and Holden, Eun-Jung,'DYNAMIC FINGERSPELLING RECOGNITION USING GEOMETRIC AND MOTION FEATURES School of Computer Science \& Software Engineering The University of Western Australia",Image Processing, 2006 IEEE International Conference on, pp. 2741-2744, 2006.

[14] Starner, Thad and Pentland, Alex,"Real-Time American Sign Language Recognition from Video Using Hidden Markov Models",pp. 227243,1997.

[15] Isaacs, Jason and Foo, Simon,'Optimized wavelet hand pose estimation for American sign language recognition",Proceedings of the 2004 Congress on Evolutionary Computation, CEC2004,pp. 797-802,vol. 1, 2004.

[16] Paulraj, M. P. and Yaacob, Sazali and Desa, Hazry and Majid, Wan Mohd Ridzuan Wan Ab,"Gesture recognition system for Kod Tangan Bahasa Melayu (KTBM) using neural network",Proceedings of 2009 5th International Colloquium on Signal Processing and Its Applications, CSPA 2009,pp. 19-22,2009.

[17] Paulraj, M. P. and Yaacob, Sazali and Desa, Hazry and Hema, C. R. and Wan Ab Majid, Wan Mohd Ridzuan,"Extraction of head and hand gesture features for recognition of sign language", 2008 International Conference on Electronic Design, ICED 2008,2008.

[18] Quan, Yang,"Chinese sign language recognition based on video sequence appearance modeling",Proceedings of the 2010 5th IEEE Conference on Industrial Electronics and Applications, ICIEA 2010,pp. 15371542,2010 .

[19] Madeo, Renata C. B. and Peres, Sarajane M. and Bíscaro, Helton H. and Dias, Daniel B. and Boscarioli, Clodis,"A Committee Machine Implementing the Pattern Recognition Module for Fingerspelling Applications",pp. 954-958, 2010.

[20] Henrique, Carlos and Monteiro, A and Felipe, Luiz and Pecoraro, Inácio and Lacerda, Angélica Takamine and Corbo, Anna Regina and Matos Araujo, Gabriel,"Um sistema de baixo custo para reconhecimento de gestos em LIBRAS utilizando visão computacional"

[21] Kleber Padovani de Souza, Jéssica Barbosa Dias, Hemerson Pistori,"Reconhecimento Automático de Gestos da Língua Brasileira de Sinais utilizando Visão Computacional", Lecture Notes in Mathematics,pp.59-95,vol. 2255,2019.

[22] Gonc, Vagner M and Peres, Sarajane M and B, Rua Arlindo and S, Av Trabalhador,'Funções de Similaridade em CBIR no Covntexto de Teste de Desempenho de Fuções de Imagens de Gestos da LIBRAS Software : Estudo de Caso em Segmentação",pp. 6, 2018. 